\title{
Liquid Crystal Phase Control Method Based on Blazed Grating Model Hongyang Guo ${ }^{1,2,3, a}$, Shengping $\mathrm{Du}^{1,2, \mathrm{~b}, *}$
}

${ }^{1}$ Key Laboratory of Optical Engineering ,Chinese Academy of Sciences,Chengdu 610209,China

${ }^{2}$ The Institute of Optics and Electronics, Chinese Academy of Sciences, Chengdu 610209, China

3University of Chinese Academy of Sciences,Beijing 100049,China

aguohongy93@163.com, bdu_shengping@163.com

${ }^{*}$ Corresponding author

Keywords: Blazed grating; Liquid crystal; Phase modulation; Diffraction intensity.

Abstract. Blazed grating, as one of the gratings, is widely used in liquid crystal phased array with beam deflection. Based on the wave control method of blazed grating model, the relationship between the beam deflection angle and the grating constant are deduced. The construction of liquid crystal phased array blazed grating model based on diffraction intensity changes with the derivation of secondary phase modulation, simulation results show that with the normalized phase difference between electrode increased, the original zeroth main diffraction will be all transferred to the first main pole, to achieve the transfer of light energy and the beam deflection.

\section{Introduction}

Blazed grating is a phase grating interference type that separates central maximum with the zero maximum in the single groove surface diffraction.It has zero order light and high diffraction efficiency . now, blazed grating has been widely used in measurement, display, communication, laser beam shaping and other fields ${ }^{[1]}$.

As a kind of blazed grating, The strongest point of the grating Fraunhofer diffraction energy transfers from level zeroth diffraction maximum to level first,currently used for blazed grating phased array model two binary optical elements based on liquid crystal phased array beam deflection ${ }^{[2]}$. Two elements refer to the surface of the glass or crystal optical material with a laser etching means two or more, the approximate continuous distribution level, so that the whole device is similar to the incident light relief structure, phase modulation, diffractive optical elements with high diffraction efficiency ${ }^{[3]}$. Blazed grating can be considered as one dimensional zigzag phase modulation grating in two element optics.

\section{Principle of blazed gating optical phased array}

The one-dimensional zigzag phase grating whose period is $T$ and the zigzag depth is $d$. The refractive index of the grating material is $n$,incident light wavelength is $\lambda$, and the optical transmission function of the one-dimensional zigzag phase grating can be written as formula (1)

$$
t(x)=\sum_{m} \delta(x-m T) * \operatorname{rect}\left(\frac{x}{T}\right) \mathrm{e}^{\mathrm{i} 2 \pi f_{0} x}
$$

In the formula(1), $f_{0}=(n-1) d / \lambda T$, the grating zigzag number is $m$, when the plane wave of unit amplitude is perpendicular to the zigzag phase grating, the transmission angular spectrum is the Fourier transform of the optical transmission function(2)

$$
\mathscr{F}[t(x)]=\sum_{m} \delta\left(f-\frac{m}{T}\right) \frac{\sin \left[\pi T\left(f-f_{0}\right)\right]}{\pi T\left(f-f_{0}\right)}
$$


When the incident light passes through the far field of the grating,the first major amplitude of Fraunhofer diffraction is expressed as function(3).

$$
A_{m}=\frac{\sin \left[\pi T\left(\frac{m}{T}-f_{0}\right)\right]}{\pi T\left(\frac{m}{T}-f_{0}\right)}
$$

The secondary diffraction intensity of $m$ is expressed as function(4)

$$
I_{m}=A_{m} \bullet A_{m}^{*}
$$

The formula(3) is brought into formula (4), and therefore $f_{0}=(n-1) d / \lambda T$, the diffraction efficiency can be written as:

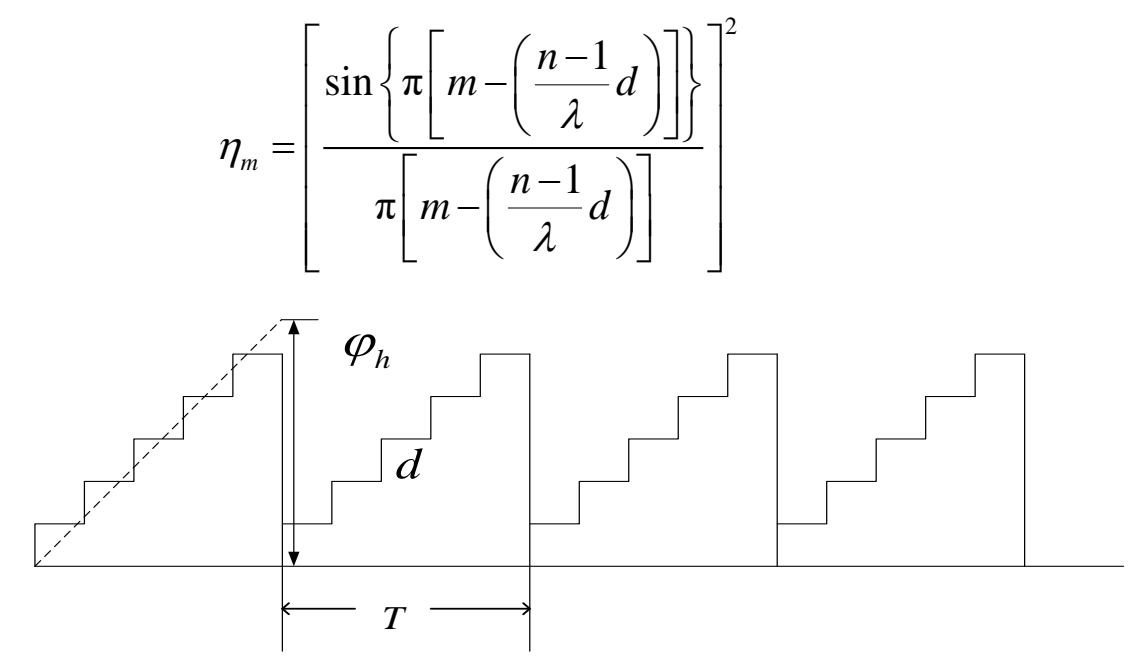

Fig.1 Multi-degree phase grating

In fact, it is difficult to make zigzag phase contours. In the two element optical technique, the outline of the zigzag phase grating can be quantized zed into step shape, as shown in figure 1. Obviously, the more the number of steps in a grating period, the smaller the quantization error, the closer to the zigzag phase grating outline.

\section{Liquid crystal phased array}

The advantages of liquid crystal is light phase modulation continuous controllable,single liquid crystal box is composed of a transparent conductive electrode and liquid crystal materials in liquid crystal electrode clamp, a single liquid crystal box is a phase control unit.Each liquid crystal cell electrode voltage controlled independently, changing the voltage of LCD electrode is controlled by the liquid crystal phase of incident light box. The incident light passing through the crystal surface of the wavefront is called multi-step phase grating periodic profile shown in Figure 2, this is a simulation of the liquid crystal grating in binary raster.

When the phase change of liquid crystal pixel unit is arranged in a ladder shape approximation of periodic blazed grating jagged. When the working wavelength is $T$, the phase is proportional to the optical path and the optical path $l=n_{e} \bullet d$, as the thickness of the liquid crystal layer is $d$, changing the refractive index will change the optical path, thus changing the phase modulation of incident light. Changing the voltage applied to the electrodes can change the liquid crystal molecules and the crystal refractive index of each pixel unit e, thus changing the light propagation in liquid crystal phase difference in each pixel unit and completing phase modulation. 


\subsection{Calculation of deflection angle of beam}

Assuming the liquid crystal blazed grating single cycle consists of liquid crystal electrodes, with appropriate voltage in the periodic grating electrode, a liquid crystal electrode to form a micro step, each step of the same height in single phase, the grating period has the largest phase delay, the liquid crystal has blazed grating period, the LCD can be equivalent to the two element model of blazed grating.

In a single grating period, each liquid crystal electrode can be seen as a modulation phase, liquid crystal grating slit width of the slit, the aperture function, liquid crystal cycle has such slit aperture function can be written as formula (6):

$$
t_{s}(x, y)=\operatorname{rect}\left(\frac{x}{a}\right) \otimes \sum_{m=0}^{M} \delta(x-m a) \exp (\mathrm{i} m \varphi)
$$

The liquid crystal surface has a plurality of grating period, grating can be regarded as a single periodic translation, liquid crystal blazed grating aperture function can be written as formula (7) ${ }^{[6]}$

$$
t(x, y)=\left[\operatorname{rect}\left(\frac{x}{a}\right) \otimes \sum_{m=0}^{M} \delta(x-m a) \exp (\mathrm{i} m \varphi)\right] \otimes \sum_{m=0}^{N} \delta(x-m D)
$$

Assuming the unit plane wave incidence, according to Fraunhofer diffraction theory, the complex amplitude and liquid crystal blazed grating exit surface complex amplitude Fourier transform relation in the far field observation screen, so the aperture function in Fourier transform far field amplitude shown as formula (8).

$$
\begin{aligned}
& \tilde{E}(u, v)=\mathscr{F}(t(x, y) \\
& =\mathscr{F}\left[\left(\operatorname{rect}\left(\frac{x}{a}\right) \otimes \sum_{m=0}^{M-1} \delta(x-m a) \exp (\mathrm{i} m \varphi)\right) \otimes \sum_{m=0}^{N} \delta(x-m D)\right] \\
& =\operatorname{sinc}(\pi a u) \frac{\sin (\pi M(a u-\varphi / 2 \pi))}{\sin (\pi(a u-\varphi / 2 \pi))} \frac{\sin (\pi N D u)}{\sin (\pi D u)}
\end{aligned}
$$

As the angle $\theta$ is the far field observation point and liquid crystal grating normal visible, applying phase shift equal phase difference is formed after the liquid crystal blazed grating built in each electrode on the diffraction grating, which is the essence of diffraction grating in multi slit interference factor in the translation axis, he let the light energy to the secondary transfer.

The complex amplitude of the far field for each secondary is obtained as formula (9)

$$
\tilde{E}(u, v)=\frac{\sin (m \pi / M)}{m \pi} \frac{\sin \pi(m-1)}{\sin \pi(m-1) M}
$$

The far field diffraction intensity can be obtained as formula (10)

$$
I=\tilde{E}(u, v) \tilde{E}(u, v)^{*}=\left[\frac{\sin (m \pi / N)}{m \pi} \frac{\sin \pi(m-1)}{\sin \pi(m-1) / N}\right]^{2}
$$

\subsection{Simulation results}

According to formula (10), the far field light intensity is simulated as figure 2 . 


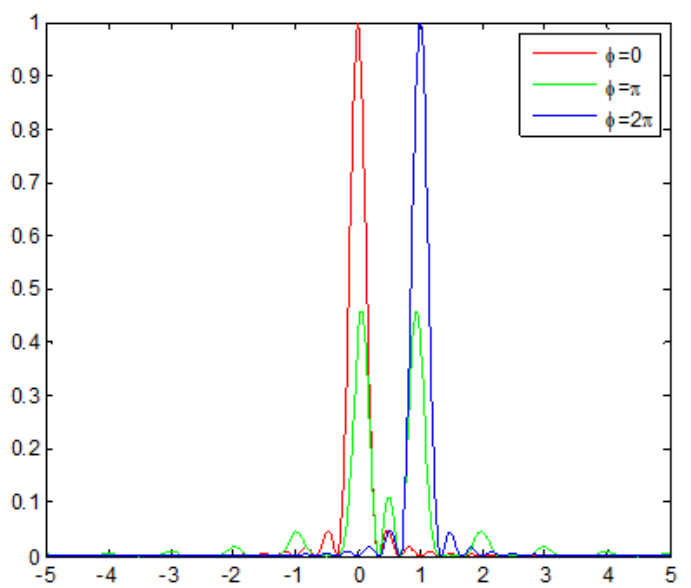

Fig. 2 Relation of secondary intensity change with phase modulation

Figure 2 is the secondary diffraction intensity changes with phase modulation relation normalization simulation, the red curve can be seen, the phase difference between the electrode and the liquid crystal grating is a diffraction grating with liquid crystal phase modulation was gradually increased, gradually transfer light energy, when the maximum modulation $\varphi_{h}=\pi$, the energy of the zeroth level diffraction major and the first main maximum is each half, continue to increase the maximum amount of phase modulation to the zeroth level, the original main diffraction maximum will all transferred to the first level main pole, achieving the transfer of light energy and the beam deflection.

\section{Summary}

Based on the wave control method of blazed grating model, the relationship between the beam deflection angle and the grating constant based on the blazed grating model and the relationship between the diffraction efficiency and the deflection angle of the beam are deduced. The construction of liquid crystal phased array blazed grating model based on diffraction intensity changes with the derivation of secondary phase modulation, simulation results show that with the normalized phase difference between electrode increased, the original zeroth main diffraction maxima will all transferred to the first level main pole, achieving the transfer of light energy and beam deflection.

\section{References}

[1] Wang X, Wilson D, Muller R, et al. Liquid-crystal blazed-grating beam deflector.. Applied Optics, vol. 39,pp.6545-55.2000.

[2] T Aoyagi, S Namba . High - efficiency blazed grating couplers. Applied Physics Letters, vol.29,pp.303-304.1976.

[3] R Kashyap, R Wyatt, R J Campbell . Wide band gain flattened erbium fibre amplifier using a photosensitive fibre blazed grating. Electronics Letters, vol. 29,pp.154-156.1993.

[4] Cai Zhang, Yongmei Huang, Bo Qi , etc. The beam deflection control based on liquid crystal spatial light modulator, Chinese laser, vol. 38,pp. 113-117,2011.

[5] Zhang Cuiyu, Huang Ziqiang. Analysis of the characteristics of two element optical liquid crystal blazed grating. Journal of optics, vol. 28,pp.1231-1235. 2008.

[6] Song Guicai, Wei Wei, Wang Xin. Theory and application of physical optics . Publishing House of Peking University, 2015. 